\title{
Decrease in Muscle Tension and Reduced Pyridine Nucleotides of the Guinea Pig Ileal Longitudinal Smooth Muscle in High $\mathrm{K}^{+}, \mathrm{Na}^{+}$-Deficient Solution
}

\author{
Kazumasa Shimizu, Tsuyoshi Kaburagi, Shinjiro Nakajyo and Norimoto Urakawa \\ Department of Veterinary Pharmacology, Nippon Veterinary and Zootechnical College, \\ Musashino, Tokyo 180, Japan \\ Received October 13, 1990 Accepted March 4, 199I
}

\begin{abstract}
In the present experiment, we studied the inhibitory mechanism of $\mathrm{Na}^{+}$ depletion on high $\mathrm{K}^{+}$-induced contraction by simultaneously measuring reduced pyridine nucleotides (PNred) or oxidized flavoproteins (FPox) fluorescence and contractile tension of the guinea pig ileal longitudinal muscle. Tension, PNred and FPox were all reversibly increased by the addition of hyperosmotic $65 \mathrm{mM} \mathrm{KCl}\left(\mathrm{H}-65 \mathrm{~K}^{+}\right)$. A high $\mathrm{K}^{+}, \mathrm{Na}^{+}$-deficient (Iso-154K ${ }^{+}$) solution induced a contraction followed by a gradual relaxation and gradually decreased PNred fluorescence. A hyperosmotic addition of $\mathrm{NaCl}$ to the Iso- $154 \mathrm{~K}^{+}$solution prevented the decreases in tension and PNred fluorescence. Addition of pyruvate or oxaloacetate restored the decrease in Iso$154 \mathrm{~K}^{+}$-induced contraction, but not the decrease in PNred fluorescence. In contrast to the PNred fluorescence, an application of the Iso- $154 \mathrm{~K}^{+}$solution increased the FPox fluorescence which was not significantly changed by an addition of $\mathrm{NaCl}$, pyruvate or oxaloacetate. These results suggest that the inhibitory mechanism of $\mathrm{Na}^{+}$depletion on the Iso- $154 \mathrm{~K}^{\dagger}$-induced contraction is an inhibition of glucose utilization.
\end{abstract}

An application of hyperosmotically added high $\mathrm{K}^{+}$solution produced a sustained contraction, while an isosmotically substituted

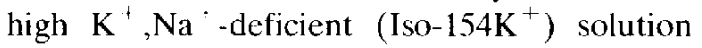
induces a contraction followed by a gradual relaxation in various smooth muscle preparations $(1-7)$. In the taenia coli (6), urinary bladder (2) and vas deferens (7), we have suggested that the inhibition of contraction by the high $\mathrm{K}^{+}, \mathrm{Na}^{+}$-deficient solution is mainly due to the inhibition of glucose utilization resulting from $\mathrm{Na}^{+}$deficiency in the medium. On the other hand, fluorometric measurements of reduced pyridine nucleotides (PNred) have been applied to isolated skeletal $(8-11)$ and cardiac muscles $(12,13)$. Recently, Ozaki et al. (14) have reported that PNred and oxidized flavoproteins (FPox) were measured fluorometrically in the intestinal smooth muscle strip of guinea pig taenia coli simultaneously with contractile tension. In the present experiment, we studied the inhibitory mechanism of $\mathrm{Na}^{+}$ depletion in the Iso-154K ${ }^{+}$-induced contraction by simultaneously measuring PNred or FPox fluorescence and contractile tension in the guinea pig ileal longitudinal muscle. 


\section{MATERIALS AND METHODS}

Male guinea pigs weighing 300 to $400 \mathrm{~g}$ were killed by a blow on the head and bled to death. After exsanguination, the abdomen was opened, and the ileum was removed. The lower part of the ileum, a $10-\mathrm{cm}$ stretch from the opening of the caecum, was discarded. The ileal longitudinal smooth muscle preparations were made as described by Paton and Aboo Zar (15). Physiological salt solution (PSS) contained: $136.9 \mathrm{mM} \mathrm{NaCl}, 5.4 \mathrm{mM} \mathrm{KCl}, 1.5 \mathrm{mM}$ $\mathrm{CaCl}_{2}, 1.0 \mathrm{mM} \mathrm{MgCl}, 11.9 \mathrm{mM} \mathrm{NaHCO} 3$, and $5.5 \mathrm{mM}$ glucose. The solution was continuously bubbled with $95 \% \mathrm{O}_{2}, 5 \% \mathrm{CO}_{2}$ at $37^{\circ} \mathrm{C}$ and $\mathrm{pH} 7.2$. High $\mathrm{K}^{+}(65.4 \mathrm{mM})(\mathrm{H}-$ $65 \mathrm{~K}^{+}$) solution was made by increasing the $\mathrm{KCl}$ concentration in the PSS. Isosmotic $154.1 \mathrm{mM} \mathrm{K}^{+}$solution was made by substituting an equimolar amount of $\mathrm{K}^{+}$for $\mathrm{Na}^{+}$in the PSS. $\mathrm{NaCl}(25 \mathrm{mM})$, pyruvate $(5.5 \mathrm{mM})$ or oxaloacetate $(5.5 \mathrm{mM})$ was added to Iso$154 \mathrm{~K}^{+}$solution hyperosmotically.

Simultaneous recordings of PNred or FPox fluorescence and force development were carried out by the method of Ozaki et al. (14). To detect PNred fluorescence, 470-nm emission wavelength and $340-\mathrm{nm}$ excitation wavelength were employed; and to detect FPox fluorescence, $530-\mathrm{nm}$ emission wavelength and $450-\mathrm{nm}$ excitation wavelength were employed. These experiments were performed with a fluorimeter specially designed to measure the fluorescence of living tissues (CAF100, Japan Spectroscopic). The muscle strip was held horizontally in a temperature controlled 7-ml volume organ bath. One end of the muscle strip was connected to a straingauge transducer to monitor the mechanical activity. The time constant of the optical channels was $0.25 \mathrm{sec}$. The fluorescence intensity obtained under the basal incubation conditions was deliberately chosen as $0 \%$ and that with $\mathrm{H}-65 \mathrm{~K}^{+}$solution, as $100 \%$ and changes were expressed as a percent of this value.
RESULTS

Changes in tension and PNred or FPox fluorescence in high $K^{+}$-treated muscles

$H-65 K^{+}$solution: An application of $\mathrm{H}-65 \mathrm{~K}^{+}$ produced a transient contraction followed by a sustained one. PNred fluorescence increased simultaneously with or immediately after the initiation of muscle contraction. When H$65 \mathrm{~K}^{+}$was removed, the fluorescence began to decrease $5-10 \mathrm{sec}$ after the initiation of relaxation, and the fluorescence was still decreasing toward the resting level when the muscle was completely relaxed (Fig. 1A). In contrast to the PNred fluorescence, the FPox fluorescence started to increase just before the initiation of the $\mathrm{H}-65 \mathrm{~K}^{+}$-induced contraction. When the muscle strip was relaxed by removal of $\mathrm{H}-65 \mathrm{~K}^{+}$, the fluorescence began to decrease after a latency of $1-5 \mathrm{sec}$ (Fig. 1B). Tension, PNred and FPox fluorescence were all reversibly increased by the addition of $\mathrm{H}$ $65 \mathrm{~K}^{+}$. On the initiation of $\mathrm{H}-65 \mathrm{~K}^{+}$-induced contraction in the ileal muscle, PNred fluorescence increased immediately after the onset of contraction, while FPox fluorescence increased before the initiation of contraction in the same manner as in taenia coli (14).

Iso-154K ${ }^{+}$solution: An application of Iso$154 \mathrm{~K}^{+}$solution induced a large contraction followed by gradual relaxation. The contraction at $20 \mathrm{~min}$ after the application of Iso$154 \mathrm{~K}^{+}$solution was $35.2 \pm 4.2 \%(\mathrm{n}=12)$ of that by $\mathrm{H}-65 \mathrm{~K}^{+}$. As shown in Fig. $1 \mathrm{~A}$, the level of PNred fluorescence (upper trace) in the presence of Iso- $154 \mathrm{~K}^{+}$solution gradually decreased to $22.2 \pm 5.2 \%(\mathrm{n}=6)$ of that by $\mathrm{H}-65 \mathrm{~K}^{+}$at $20 \mathrm{~min}$. At $20 \mathrm{~min}$ after treatment with the Iso- $154 \mathrm{~K}^{+}$solution, the maximum level of the FPox fluorescence was $98.5 \pm$ $3.2 \%(\mathrm{n}=6)$ of that by $\mathrm{H}-65 \mathrm{~K}^{+}$(Fig. 1B). Because an exchange of Iso- $154 \mathrm{~K}^{+}$solution with the PSS caused a small stimulus artifact and the fluorescence change comes very fast, the initiation of change in PNred or FPox fluorescence was unclear in the Iso- $154 \mathrm{~K}^{+}$. treated muscle. 

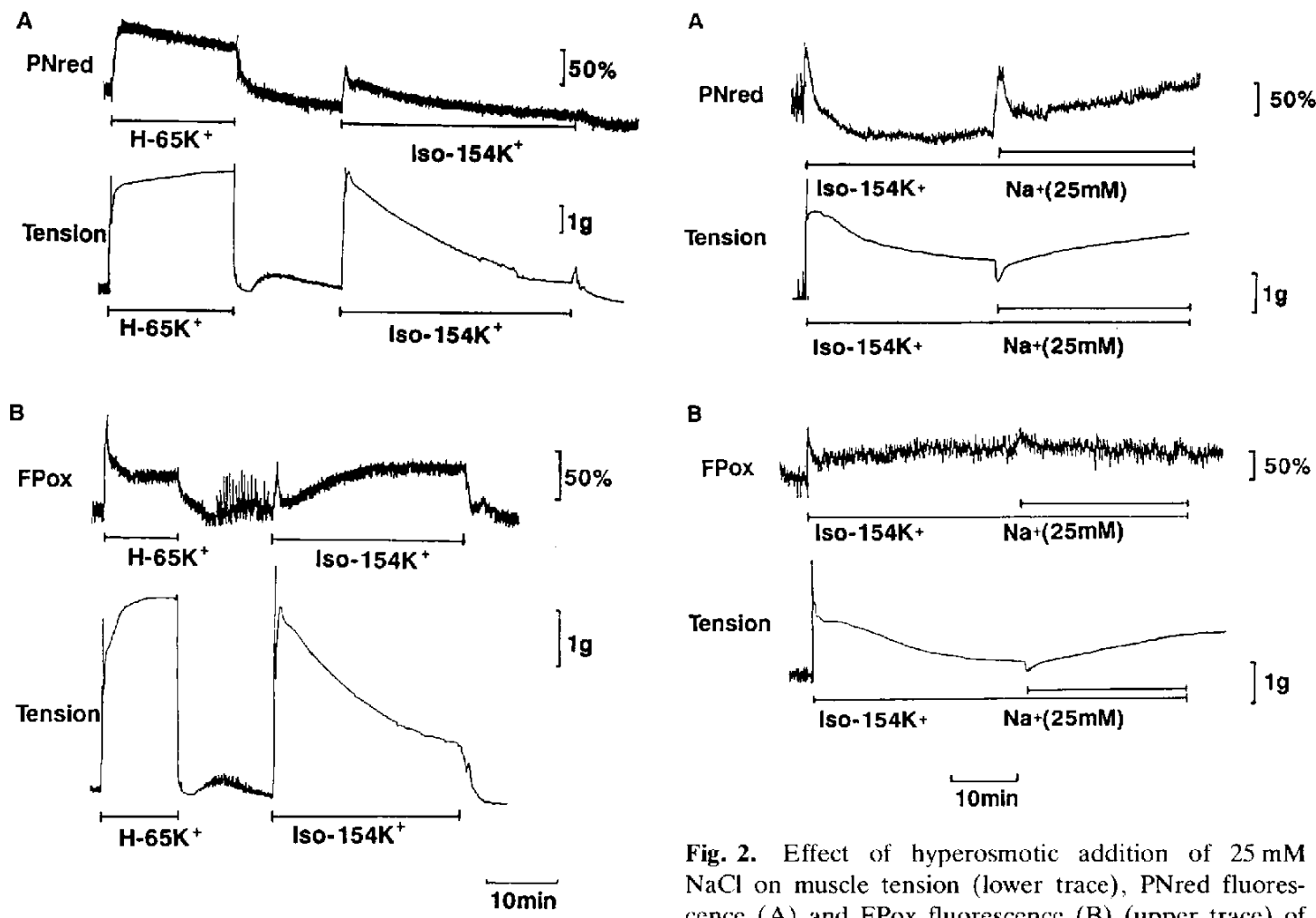

Fig. 2. Effect of hyperosmotic addition of $25 \mathrm{mM}$ $\mathrm{NaCl}$ on muscle tension (lower trace), PNred fluorescence (A) and FPox fluorescence (B) (upper trace) of the ileal muscle in $I$ so- $154 \mathrm{~K}^{+}$solution. After the changes in fluorescence and tension of the ileal muscle reached constant levels in the presence of Iso- $154 \mathrm{~K}^{+}$ solution, $25 \mathrm{mM} \mathrm{Na}^{+}$was applied.

Fig. 1. Changes in PNred fluorescence (A), FPox fluorescence (B) and muscle contraction in the ileal muscle stimulated by hyperosmotically added $65.4 \mathrm{mM}$ $\mathrm{K}^{+}\left(\mathrm{H}-65 \mathrm{~K}^{+}\right)$or isosmotically added $154.1 \mathrm{mM} \mathrm{K}^{+}$ (Iso-154K ${ }^{+}$) solution. After the treatment, H-65K ' or Iso- $154 \mathrm{~K}^{+}$solution was exchanged with the normal solution. An artifact of the solution exchange disturbed a transient rise of fluorescence level of PNred or FPox. The vertical bars present the percent change in fluorescence, taking the basal fluorescence as $0 \%$ and that with $\mathrm{H}-65 \mathrm{~K}^{+}$solution as $100 \%$.

Effect of a hyperosmotic addition of $\mathrm{Na}^{1}$ to the 1 so- $154 \mathrm{~K}^{+}$treated muscle

The decrease in tension induced by the Iso$154 \mathrm{~K}^{+}$solution in the ileal muscle was gradually reversed by a hyperosmotic addition of $\mathrm{Na}^{+}$at a concentration of $25 \mathrm{mM}$ (Fig. 2A). When $25 \mathrm{mM} \mathrm{Na}^{+}$was applied to the muscle, the decrease in PNred fluorescence due to Iso$154 \mathrm{~K}^{+}$solution was gradually restored, although FPox fluorescence was not affected (Fig. 2B). The concentration-response relationship between $\mathrm{Na}^{+}$concentration and muscle tension or PNred fluorescence of the

tissue in $\mathrm{Iso}-154 \mathrm{~K}^{+}$solution is shown in Fig. $3 \mathrm{~A}$. Increasing $\mathrm{Na}^{+}$concentration induced an increase in the tension or the level of PNred fluorescence. A correlation was obtained between the tension development and the PNred fluorescence (Fig. 3B). The regression line calculated from the data was $\mathrm{Y}=1.2 \mathrm{X}-23.3$ with a correlation index $(\gamma)$ of 0.997 .

Effect of adding pyruvate or oxaloacetate to the Iso- $154 \mathrm{~K}^{+}$treated muscle

It is well-known that the sustained contraction induced by high $\mathrm{K}^{+}$solution in guinea pig intestine is dependent on the external glucose in the presence of $\mathrm{Na}^{+}$. Both pyruvate and oxaloacetate, substrates for oxidative metabolism, are as effective as glucose on the contraction even in the absence of $\mathrm{Na}^{+}$(6). When the muscle strip was relaxed by the sub- 
A

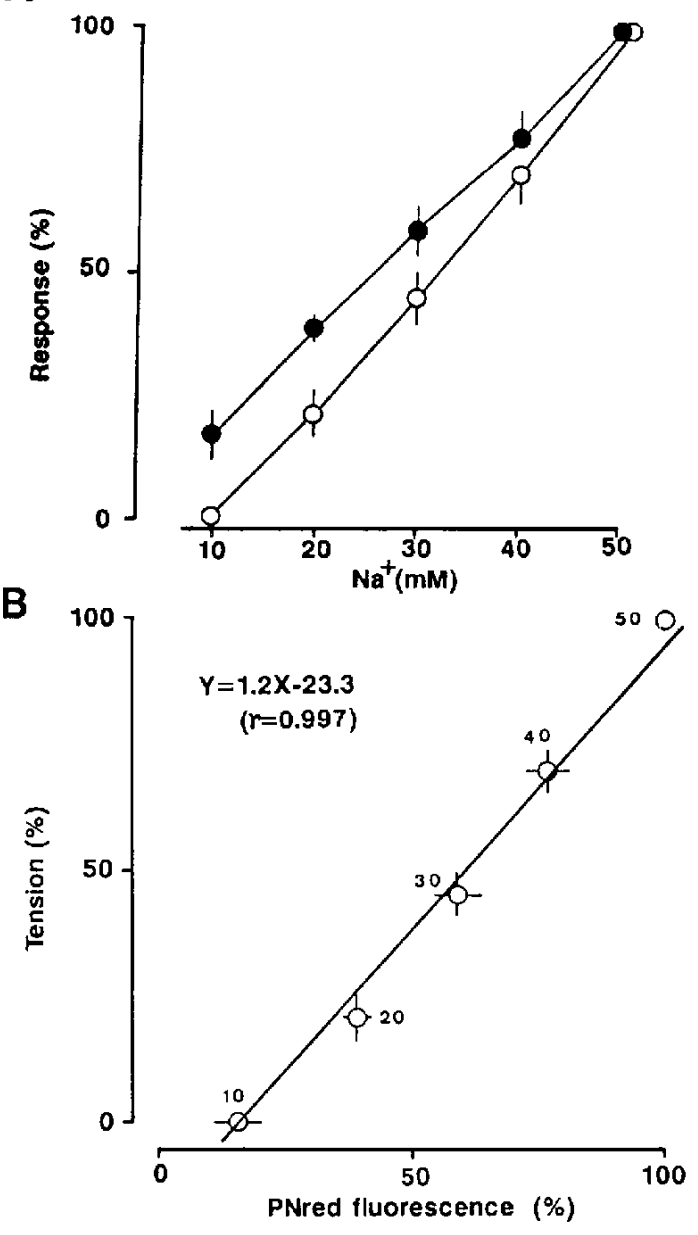

Fig. 3. Effect of external $\mathrm{Na}^{+}$concentration on PNred fluorescence and tension development of the ileal longitudinal muscle in the Iso- $154 \mathrm{~K}^{+}$solution. (A) Effect of cumulative application of $\mathrm{Na}^{+}$on PNred fluorescence (O) and muscle tension (O). The muscle strip was treated with Iso- $154 \mathrm{~K}^{+}$solution for $20-30$ min. Responses induced by an application of $50 \mathrm{mM}$ $\mathrm{Na}^{+}$were taken as $100 \%$. (B) Correlation between PNred fluorescence and tension development in the presence of different concentration of $\mathrm{Na}^{+}$. The $\mathrm{Na}^{+}$ concentration was changed between 10 and $50 \mathrm{mM}$, as indicated by the number in the Figure.

stitution of Iso- $154 \mathrm{~K}^{+}$solution, addition of 5.5 $\mathrm{mM}$ pyruvate restored the tension to $95.4 \pm$ $3.8 \%(\mathrm{n}=10)$ of that by $\mathrm{H}-65 \mathrm{~K}^{\dagger}$, but not the PNred fluorescence, although the level of FPox fluorescence in the presence of Iso$154 \mathrm{~K}^{+}$solution plus pyruvate $(5.5 \mathrm{mM})$ was
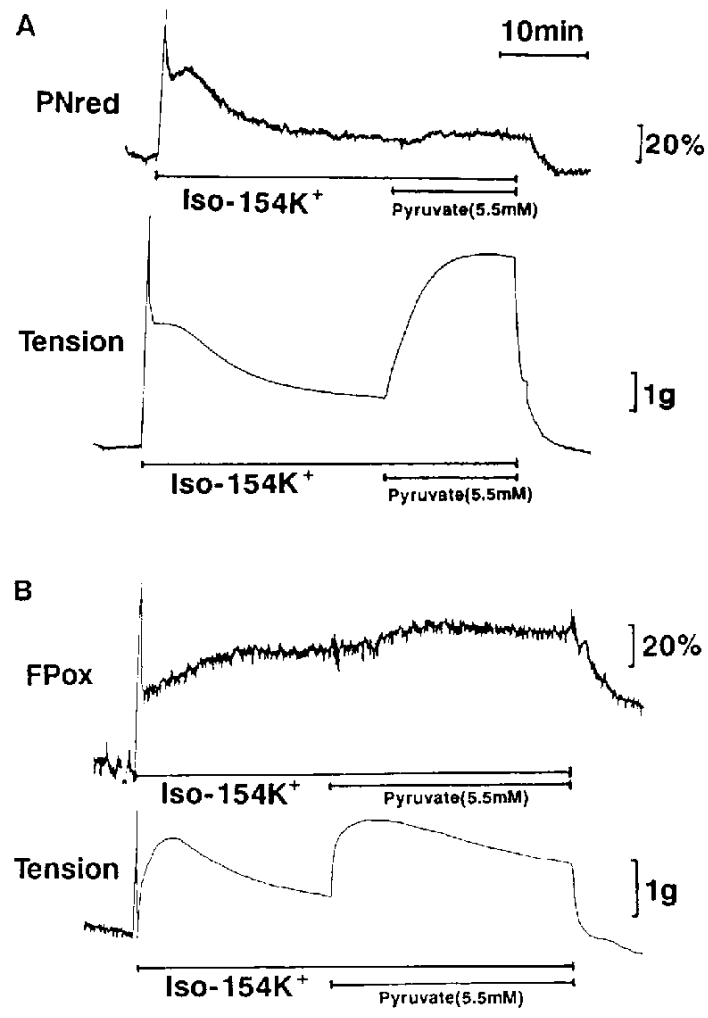

Fig. 4. Effect of $5.5 \mathrm{mM}$ pyruvate on muscle tension (lower trace). PNred fluorescence (A) and FPox fluorescence (B) (upper trace) of the ileal muscle in Iso- $154 \mathrm{~K}^{+}$solution. The PSS was replaced with Iso$154 \mathrm{~K}^{+}$solution and then $5.5 \mathrm{mM}$ pyruvate was added.

$96.4 \pm 4.9 \%(\mathrm{n}=4)$ of that by $\mathrm{H}-65 \mathrm{~K}^{1}$, which is similar to those in Iso- $154 \mathrm{~K}^{+}$solution (98.5 \pm 3.2\%, $\mathrm{n}=6$ ) (Fig. $4, \mathrm{~A}$ and B). On the changes in muscle tension, PNred or FPox fluorescence, oxaloacetate $(5.5 \mathrm{mM})$ showed similar effects to those of pyruvate (Fig. 5, A and B).

\section{DISCUSSION}

Fluorescence techniques have been applied to measurements of the redox state of PNred in a number of intact tissue preparations. Skcletal muscle was the first tissue in which this method was successfully applied (8), and intensive fluorometric studies helped to elucidate the energy metabolism of the heart muscle $(12,13)$. On the other hand, Ozaki et al. 

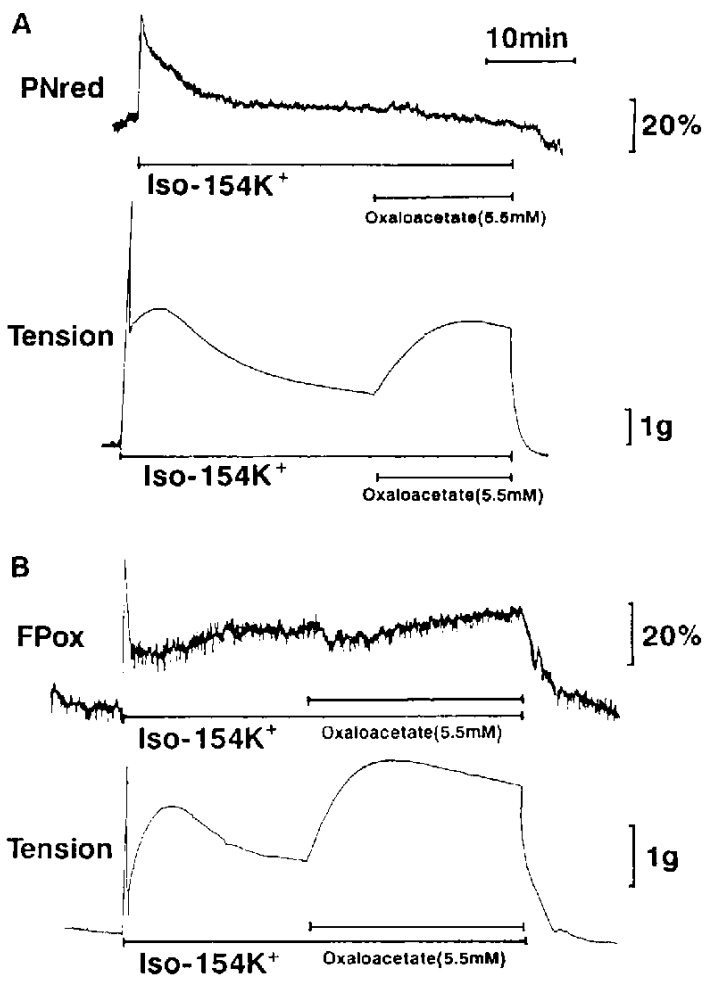

Fig. 5. Effect of $5.5 \mathrm{mM}$ oxaloacetate on muscle tension (lower trace), PNred fluorescence (A) and FPox fluorescence (B) (upper trace) of the ileal muscle in Iso- $154 \mathrm{~K}^{+}$solution. The PSS was replaced with Iso$154 \mathrm{~K}^{+}$solution, and then $5.5 \mathrm{mM}$ oxaloacetate was added.

(14) have reported that PNred and FPox were measured fluorometrically in the intestinal smooth muscle of guinea pig taenia coli simultaneously with contractile tension. That is, in the $\mathrm{K}^{+}$-depolarized muscle, the increase in PNred was decreased when glycolysis was inhibited by removing glucose and adding 2-deoxyglucose, but not when oxidative metabolism was inhibited by $\mathrm{N}_{2}$ bubbling or by $\mathrm{NaCN}$. In contrast to this, the increase in the FPox by $\mathrm{K}^{+}$-depolarization was decreased by $\mathrm{N}_{2}$ bubbling or $\mathrm{NaCN}$, but not by the inhibition of glycolysis. From these results, they have suggested that the major portion of PNred measured fluorometrically in their study exists in the cytoplasm and the amount is regulated by glycolysis.

It is known that hyperosmotically added high $\mathrm{K}^{+}$induces a contraction in the intestinal smooth muscle, which is maintained at a high and steady level but inhibited by glucose removal, suggesting that the contraction is supported by an energy supply from aerobic glycolytic metabolism $(16-18)$. On the other hand, a high $\mathrm{K}^{+}, \mathrm{Na}^{+}$-deficient solution induces another type of contraction which is followed by a gradual decline in tension level. The decreased tension is restored by the addition of $\mathrm{Na}^{+}$to the medium containing glucose or by the addition of pyruvate or oxalacetate which can penetrate through the cell membrane without external $\mathrm{Na}^{+}$. From these data, we have proposed that $\mathrm{Na}^{+}$deficiency in high $\mathrm{K}^{+}$solution inhibits the contraction due to inhibition of glucose utilization in the muscle (6). From the biochemical approach, $\mathrm{O}_{2}$ consumption and ATP content of the muscle was measured during the $154 \mathrm{~K}^{+}$-induced contraction. The $\mathrm{Na}^{+}$-deficiency decreased the increased $\mathrm{O}_{2}$ consumption and ATP content of the muscle treated with high $\mathrm{K}^{+}(18)$. However, these measurements were performed at only one or two points of the experimental procedure. In the present experiments, we simultaneously recorded the changes in the muscle tension and PNred or FPox fluorescence during the whole course of the experiment in the ileal muscle.

An application of Iso- $154 \mathrm{~K}^{+}$solution to the tissue induced a large contraction, then gradually decreased the muscle tension, and it remarkably decreased the PNred fluorescence. This decrease in both the tension and PNred fluorescence were prevented by the hyperosmotic addition of $\mathrm{NaCl}$, but not affected by the substitution of pyruvate or oxaloacetate for glucose in Iso- $154 \mathrm{~K}^{+}$solution. On the other hand, an application of Iso- $154 \mathrm{~K}^{+}$solution to the tissue increased FPox fluorescence, which was not influenced by hyperosmotic addition of $\mathrm{NaCl}$, whereas the contraction was markably restored. Moreover, addition of 5.5 $\mathrm{mM}$ pyruvate or oxaloacetate did not significantly change the FPox fluorescence of the muscle in Iso- $154 \mathrm{~K}^{+}$solution. The reason why pyruvate or oxaloacetate does not increase 
FPox fluorescence of the tissue in Iso- $154 \mathrm{~K}^{+}$ solution is assumed to be as follows: this FPox fluorescence has been kept at a maximal level by a compensatory effect on glycolysis inhibition that is represented by the decreased in PNred fluorescence of the muscle in Iso$154 \mathrm{~K}^{+}$solution; and accordingly, pyruvate or oxaloacetate can not induce any increase in FPox fluorescence under this condition. This assumption is probably supported by the data reported by Paddle (19) that pyruvate had no effect on FPox fluorescence in the rat diaphragm when it was added under conditions in which glycolysis was inhibited.

It is well-known that intracellular $\mathrm{Ca}^{+}$ion $\left(\left[\mathrm{Ca}^{2+}\right]_{\mathrm{i}}\right)$ regulates tension development and metabolic processes in intestinal smooth muscles. To assess the possibility that a change in $\left[\mathrm{Ca}^{2+}\right]_{i}$ is involved to the relaxation of the ileal muscle in Iso- $154 \mathrm{~K}^{+}$solution, we measured simultaneously muscle tension and $\left[\mathrm{Ca}^{2+}\right]_{i}$ level using fura- 2 by the method devised by Ozaki et al. (14). In fura 2-loaded muscle strips, application of Iso- $154 \mathrm{~K}^{+}$ solution increased tension and $\left[\mathrm{Ca}^{2+}\right]_{i}$ level, followed by a gradual relaxation without a decrease in $\left[\mathrm{Ca}^{2+}\right]_{i}$ level (K. Shimizu et al., unpublished data). Therefore, the inhibition of Iso- $154 \mathrm{~K}^{+}$-induced contraction is probably not limited by the change in $\left|\mathrm{Ca}^{2+}\right|_{i}$ level.

In Fig. 3 ( $A$ and $B$ ), it was shown that the muscle tension and PNred fluorescence of the muscle in high $\mathrm{K}^{+}$solution are dependent on the external $\mathrm{Na}^{+}$concentration and that the muscle tension has a very close relationship to the PNred fluorescence at all the $\mathrm{Na}^{+}$ concentrations in high $\mathrm{K}^{+}$solution.

From these data, it is suggested that the $\mathrm{Na}^{+}$deficiency inhibits the glucose symport through the cell membrane, resulting in a decrease in an intermediate of the glycolytic pathway, PNred, in the cytoplasm. Accordingly, these results probably confirm our proposal that the decrease in the developed tension in Iso- $154 \mathrm{~K}^{+}$solution is caused by the inhibition of glucose utilization which involves a $\mathrm{Na}^{+}$ deficiency, as determined by mcasuring the change in PNred.

\section{REFERENCES}

1 Urakawa, N., Karaki, H. and Ikeda, M.: ${ }^{15} \mathrm{Ca}$ up take and tissue $\mathrm{Ca}$ of guinea pig taenia coli in isotonic high-K/Na-deficient medium. Japan. J. Pharmacol. 18, 294-298 (1968)

2 Shimizu, K., Yaguchi, S., Kometani, A., Nakajyo, S., Ozaki, H. and Urakawa, N.: A comparative study on the contraction induced by high $K / N_{a}$ deficient solution in the trachea, gall bladder and urinary bladder in guinea pig. Japan. J. Pharmacol. 34, $231-239$ (1984)

3 Suzuki, T., Karaki, H, and Urakawa, N.: Inhibition of contraction by swelling of vascular smooth muscle in high $\mathrm{KCl}$, low $\mathrm{Na}$ solution. Arch. Int. Pharmacodyn. Ther. 250, 195-203 (1981)

4 Ueda, F., Kishimoto, T., Karaki, H. and Urakawa, N.: High $\mathrm{K}$-induced contraction in rabbit and monkey tracheal smooth muscle. Japan. J. Smooth Muscle Res. 19, 541 - 549 (1983)

5 Kamizaki, I., Shimizu, K., Nakajyo, S. and Urakawa, N.: A comparative study on the contraction induced by high $\mathrm{K}^{\prime} / \mathrm{Na}^{\dagger}$ deficient solution in rat uterus or urinary bladder, Japan. J. Pharmacol. 47, $179-188$ (1988)

6 Suzuki, T., Karaki, H. and Urakawa, N.: Mechanism of inhibition of contraction by high K. Na deficient solution in smooth muscle of guinca pig taenia coli. Arch. Int. Pharmacodyn. Ther, 248, $43-49$ (1980)

7 Shimizu, K., Tahara, K., Nakajyo, S and Urakawa, N.: Effects of the high $\mathrm{K}^{+} / \mathrm{Na}^{+}$deficient solution on mechanical response and wet weight of tissue in vas deferens and seminal vesicle in guinea pig. Japan. J. Smooth Muscle Res. 24, 13-21 (1988)

8 Chance, B. and Jobsis, F.F.: Changes in fluorescence in a frog sartorius muscle following a twitch. Nature 184, 195-196 (1959)

9 Godfraind-de Becker, A.: Heat production and fluorescence changes of toad sartorius muscle during aerobic recovery after a short tetanus. J. Physiol. (Lond.) 223, $719-734$ (1972)

10 Jobsis, F.F. and Duffield, J.C.: Oxidative and glycolytic recovery metabolism in muscle. Fluorometric observations on their relative contributions J. Gen. Physiol. 50, 1009 - 1047 (1967)

11 Wendt, I.R. and Chapman, J.B.: Fluorometric studies of recovery metabolism of rat fast and slow twitch muscles. Am. J. Physiol. 230, 1644-1649 (1976)

12 Rubanyi, G. and Kovach, A.G.B.: Calcium-linked adjustment of myocardial metabolism to changing mechanical demands in the isolated rat heart. 
Acta Physiol. Acad. Sci. 55, 335 - 343 (1980)

13 Katz, L.A., Koretsky, A.P. and Balaban, R.S.: Respiratory control in the glucose perfused heart. $A{ }^{31} \mathrm{P}$ NMR and NADH fluorescence study. FEBS Lett. 221, $270-276$ (1987)

14 Ozaki, H., Satoh, T., Karaki, H. and Ishida, Y.: Regulation of metabolism and contraction by cytoplasmic calcium in the intestinal smooth muscle. J. Biol. Chem. 263, $14074-14079$ (1988)

15 Paton, W.D.M. and Aboo Zar, M.: The origin of acetylcholine released from guinea pig intestine and longitudinal muscle strips. J. Physiol, (Lond.) 194, 13-33 (1968)

16 Urakawa, N. and Holland, W.C.: ${ }^{45} \mathrm{Ca}$ uptake and tissue calcium in $\mathrm{K}^{+}$-induced phasic and tonic con- traction in taenia coli. Am. J. Physiol. 207, 873876 (1964)

17 Pfaffman, M., Urakawa, N. and Holland, W.C.: Role of metabolism in $\mathbf{K}$-induced tension changes in guinea pig taenia coli. Am. J. Physiol. 208, 1203 - 1205 (1965)

18 Karaki, H., Suzuki, T., Urakawa, N., Ishida, Y. and Shibata, $\mathrm{S}$ :: High $\mathrm{K}^{+}, \mathrm{Na}{ }^{\prime}$-deficient solution inhibits tension, $\mathrm{O}_{2}$ consumption and ATP synthesis in smooth muscle. Japan. J. Pharmacol. 32, $727-733$ (1982)

19 Paddle, B.M.: A cytoplasmic component of pyridine nucleotide fluorescence in rat diaphragm: evidence from comparisons with flavoprotein fluorescence. Pflugers Arch. 404, 326-331 (1985) 